\title{
La psicoterapia como una construcción conjunta del sentido de realidad. Un acercamiento a los principios constituyentes de una interacción psicoterapéutica
}

\section{Psychotherapy as a joint construction of the sense of reality. An approach to the constituent principles of a psychotherapeutic interaction}

\author{
Cristóbal Pacheco \\ Universidad Autónoma de Chile, Chile \\ Pablo Fossa \\ Universidad del Desarrollo, Chile \\ (Rec: agosto de 2018 - Acept: mayo de 2019)
}

\begin{abstract}
Resumen
El desarrollo de una intervención psicoterapéutica compromete un encuentro interaccional, caracterizado por la necesaria conjunción de multiversos que convergen en una esfera dialógica, asociada a la resolución de una problemática o consecución de objetivos por parte de la figura consultante. Es en esta interacción dialógica, donde surgen las dudas sobre si la psicoterapia responde a un encuentro de miradas o bien, a una co-construcción de un sentido de realidad entre terapeuta y consultante, miradas que representan un posible descarte o complementariedad en su propia constitución.

Una postura sobre la interacción en psicoterapia necesita establecer una posición activa respecto del rol del terapeuta, nutrida de una visión ontológica que repercuta directamente en un trabajo de interacción discursiva. Asimismo, estaría orientada hacia un propósito metódico que configure los quehaceres de los participantes, como elementos en continuo desarrollo, mas no en una respectiva predefinición. El presente artículo tiene como objetivo desarrollar una propuesta de modelo de integración comunicativa, resultando una oportunidad de convergencia en el encuentro de universos desde una dimensión lingüística de interacción en el desarrollo de un proceso psicoterapéutico.
\end{abstract}

Palabras clave: Interacción dialógica, encuentro de miradas, co-construcción de realidad, interacción terapéutica, visión ontológica.

\begin{abstract}
The development of psychotherapeutic intervention engages an interactional encounter, characterized by the necessary conjunction of multiverses that converge in a dialogical sphere associated with the resolution of a problem or achievement of objectives by the consulting figure. It is in this dialogical interaction, where doubts arise as to whether psychotherapy responds to a meeting of views or, to a co-construction that comprises a sense of reality between therapist and consultant, views that represent a possible discard or complementarity in its own nature. An approach to the interaction in psychotherapy raises the need to establish an active position regarding the role of the therapist, nourished by an ontological vision that directly affects the constitution of a work of discursive interaction. In addition, this position should be oriented towards a methodical purpose that configures the tasks of participants, not as a respective predefinition but as elements in continuous development. The present article aims to propose a communicative integration model, resulting in an opportunity for convergence in the meeting of universes from a linguistic dimension of interaction in the development of a psychotherapeutic process.
\end{abstract}

Keywords: Dialogical interaction, encounter of views, co-construction of reality, therapeutic interaction, ontological vision

Correspondencia a: Cristóbal Pacheco, cristobalp@live.cl 


\section{Introducción}

La actual situación a nivel de posturas sobre la intervención psicoterapéutica se asocia a un periodo de transición, donde emerge el elemento dialógico como una herramienta basada en la aplicación del acto comprensivo, lo cual es propio del desarrollo de un método hermenéutico (Mook, 2010). Este permite un encuentro con las posibilidades comprensivas arrojadas en las dimensiones lingüísticas que permanecen en los terrenos de las interacciones dialógicas. En este contexto, es menester establecer un planteamiento respecto al fenómeno de "fusión de horizontes" expuesto por Gadamer (2010), traduciéndolo como un encuentro entre dos perspectivas, dos multiversos que presentan construcciones particulares, movimientos históricos, culturales y percepciones de realidad distintivas, siendo una característica propia de un proceso de intervención psicoterapéutica, este relativo encuentro de miradas entre terapeuta y consultante.

Desde una perspectiva crítica y de acuerdo a los planteamientos anteriormente referidos por Gadamer, surge además lo establecido por Hans - Robert Jauss (1977), asociado al "horizonte de expectativas". Esto, desde una corriente literaria, puede comprenderse como una postura asociada a las expectativas que tanto terapeuta como consultante esperan encontrar en el desarrollo de un proceso particular, intervención que puede llevar al desarrollo de posibles "decepciones" o "frustraciones" para ambos. Es a partir de estas visiones, que la instancia de encuentro de miradas repercute de manera dramática en la forma y en la posición en la cual se puede desarrollar un proceso terapéutico, considerando la posible imposibilidad de una "conexión genuina" a nivel comunicativo. Esta se basa en el concepto de indisponibilidad del lenguaje propuesto por Gadamer (1982), el cual expone la dificultad de aislar los sesgos o preconcepciones en el desarrollo de un ejercicio hermenéutico, en vista de que estos mismos presentan un carácter imperceptible para los actores de una interacción lingüística.

Los planteamientos anteriormente expuestos, plantean el desafío de visualizar y establecer una alternativa de acto de "comunión lingüística estratégica", la cual permita que en un vínculo psicoterapéutico se establezca una cadena de vinculación dialógica con un sentido de mutua retroalimentación e intencionalidad. Desde esta perspectiva, la oportunidad de la co-construcción del sentido de realidad, propuesta desde el construccionismo social de Kenneth Gergen (2007), supone una posibilidad para la creación conjunta de un universo compartido a partir de una interacción dialógica, basada en el sentido de realidad como un sustrato socialmente construido.

El presente artículo, pretende desarrollar una visión integrada que incorpore diversos horizontes de miradas epistemológicas, a modo de elaborar un modelo de interacción terapéutica que permita graficar la relevancia del encuentro de subjetividades desde una postura de interacción dialógica.

\section{1.-Un encuentro de multiversos}

Desde una visión basada en el establecimiento de una representación cósmica y biológica de la realidad (o mejor dicho de las "realidades humanas"), surge la necesidad de emplear el concepto de multiversos, el cual es inicialmente propuesto por Humberto Maturana (1988). Este autor, establece la existencia de múltiples sentidos de realidad, los cuales dependerán netamente de la percepción entregada por la figura de un obser- vador. De acuerdo a Maturana (1997), una concepción objetiva del concepto de "realidad", implica una definición compleja si se considera la construcción perceptual; en efecto, en relación al rol de la objetividad en la percepción, el autor establece dos dimensiones de la misma: la objetividad entre paréntesis y la objetividad sin paréntesis.

Respecto a la objetividad sin paréntesis (asociada al concepto de ontologías trascendentales), se postula que la realidad ocurre con independencia de la función del observador, la validación de esta verdad externa al individuo no depende de su juicio o poder argumentativo para generar una construcción per se (Kenny, 1989). En relación a la objetividad entre paréntesis, se puede comprender de acuerdo a Maturana (1978) como una característica propia de los sistemas vivos, como organismos con un determinismo estructural con la capacidad de generar una construcción respecto a la percepción que realizan de su entorno, lo cual se asocia a la construcción de realidad desde una perspectiva intrínseca, aspecto que determina en cierta medida la definición de "la verdad individual" y su mera representación a nivel particular.

En espacios asociados a un encuentro terapéutico, se percibe una variación de la postura "privilegiada" de la figura del terapeuta, quien en cierta medida contaba con una posición de dominio teórico que le permitía una mayor capacidad de decisión respecto al concepto de verdad u objetividad. Es en esta nueva dimensión, donde la posición se invierte y el universo o concepto de realidades particulares del consultante, se encuentra con las construcciones o sesgos propios de la figura interventora, siguiendo una línea lingüística como reflejo de un acoplamiento estructural ontogénico (Maturana \& Kurt, 1994).

Al exponer las posibilidades de múltiples realidades, tanto como verdades convergen en la constitución de multiversos, solo se puede llegar a un punto de encuentro y coexistencia mediante la interacción lingüística, la cual demanda consenso a modo de lograr un conocimiento común, el cual presenta un cariz de "pacto" establecido por universos individuales (Maturana, 1987). El problema surge en las posibilidades reales de transferencias de información, considerando que estamos ante el encuentro de dos observadores, cuyas realidades se encuentran sujetas a condiciones constitutivas de carácter estructural, siendo que el elemento lingüístico como medio de transferencia de realidad, se ve enfrentado a la odisea de la transmisión vivencial existencial, dimensión que no cuenta con una posible sistematización, no siendo factible mediante el conocimiento común, lograr una interacción satisfactoria en dicha esfera (Kenny, 1989).

Ante la problemática expuesta, asociada a la imposibilidad de transmisión de elementos vivenciales en la interacción lingüística (la cual es visualizada como una alternativa de pacto y de sentido común más que como un encuentro que anule toda objetividad), surge la necesidad de recobrar un sentido que se aleje del tecnicismo adicionado a las condiciones de interacción lingüísticas. Desde Maturana (1997), es posible referir la importancia de validar, lo que el autor refiere como función del cientista humano en el encuentro con una realidad que trascienda la necesidad de explicación. Maturana (1997) propone cuatro medios de validar lo que él denomina "praxis del vivir" como conocimiento objetivo, lo cual permite a su vez brindar una validación y lograr sin duda, inmersión en la 
experiencia particular vivenciada por la figura de un interlocutor. Estos cuatro momentos o condiciones se definen de la siguiente forma:

La especificación del fenómeno, que ha de ser explicado como una característica de la praxis del vivir del observador, a través de la descripción de lo que él o ella debe hacer para experimentarlo.

La proposición en la praxis del vivir del observador de un mecanismo que, como una consecuencia de su operación, producirá en él o ella la experiencia del fenómeno por explicar.

La deducción desde el mecanismo propuesto en (b) y de todas las coherencias operacionales que este supone en la praxis del vivir del observador, de otro fenómeno, así como de las operaciones que el observador debe hacer en su praxis del vivir para experimentarlo.

La experimentación por parte del observador de aquellos fenómenos adicionales deducidos en (c), en la medida que él o ella ejecuta en su praxis del vivir aquellas operaciones que, de acuerdo con lo que han sido también deducidas en (c), serían generadas en ella cuando él o ella las realiza.

Se estima, a partir de estas condiciones, generar un espacio de comprensión a nivel interpersonal, que estime un encuentro de múltiples realidades que -desde una dimensión vivencial- puedan llegar a influirse de manera mutua. Pese a esto, la vinculación con sentido, basada en las potencialidades de un encuentro con énfasis en lo vivencial por sobre lo teórico, representa un desafío en desarrollo, lo cual es parte constituyente de la necesidad de estructuración del presente artículo.

\section{2.-La conformación del sentido de realidad}

La realidad y su conformación constituyen un principio articulador en la construcción del pensamiento científico y en particular, en el pensamiento psicológico. Desde los trabajos de Oswald Külpe, en los inicios de la disciplina, se aprecia una tensión sobre qué es la realidad y cómo se constituye en la experiencia psicológica individual y relacional (Fossa, 2018).

Desde la perspectiva socio-constructivista se puede concebir la realidad como "internalización transformadora". Este concepto acuñado por Vygotsky (1934) y posteriormente desarrollado por Valsiner en la actualidad (Valsiner \& Van der Veer, 2000) implica la manera en que los procesos sociales son internalizados en la experiencia psicológica durante el desarrollo humano, transformando así todo el funcionamiento mental y construyendo de esta manera la propia realidad para el sujeto. Un ejemplo de esto es la adquisición del lenguaje, que desde esta perspectiva es primero experimentado socialmente y luego, internalizado para ser utilizado en diversas funciones psicológicas y así construir la realidad en la consciencia. El impacto del ambiente social y cultural es vivido como una experiencia comunicativa en la consciencia a partir de significados que fueron internalizados y aprendidos, primero socialmente en la interacción con los otros.

Lo anterior quiere decir que la realidad se construye a través de procesos sociales internalizados que modifican y reorganizan toda la experiencia psicológica. El lenguaje, como un ejemplo de esto, es aprendido socialmente e internalizado, permitiendo pensar la propia experiencia y mostrando a la propia consciencia individual sus posibilidades y los límites que este mismo establece. Es así como nuestra realidad en la consciencia está determinada y limitada por las posibilidades que el lenguaje nos entrega.

Esta perspectiva nos permite pensar en la diferenciación que se ha realizado en Psicología sobre las dimensiones de sentido y significado (Cornejo, 2012). El significado como perspectiva asociacionista del lenguaje (palabra $=$ cosa) tiene relación con lo que la palabra representa. Por otro lado, el sentido como dimensión interna, tiene que ver con todo lo que la palabra "remueve" en la consciencia individual. Es ahí donde una palabra cobra sentido en algunos contextos y no otros. $O$ bien, una palabra cobra sentido para unas personas y no en otras. El impacto de la palabra en la consciencia es la construcción de la realidad. Es decir, la influencia estética de la cultura, la sociedad y el otro como alteridad remueven ciertos elementos de la consciencia, los cuales hacen construir una realidad subjetiva para sí mismo. Este proceso aumenta su complejidad cuando lo llevamos al plano relacional. En una interacción humana - por ejemplo, la relación terapéutica - los significados entregados al campo dialógico remueven elementos similares y/o diferentes en cada consciencia individual, generando así la experiencia relacional y co-construyendo una realidad compartida para el campo de relación, a partir de los significados que se remuevan en cada consciencia.

Lo anteriormente desarrollado se vincula con los planteamientos de Wittgenstein (1953) sobre los juegos de lenguaje. El lenguaje entonces, en el espacio relacional, es para alguien y en un contexto determinado. El significado y sentido de la palabra varía según la persona, el contexto y el tiempo, por lo que la realidad cobra sentido solo en un contexto específico (por ejemplo, la interacción terapéutica) y para esos sujetos, según su historia individual y la historia particular de la relación. El campo de significados compartidos y la realidad se co-construye a través de estos juegos de lenguaje particulares de la relación y en el marco de esa consciencia colectiva.

Para comprender la noción del sentido de realidad entonces es importante comprender la noción de historicismo. La noción de sujetos históricos determina la construcción y co-construcción de la realidad (Vygotsky, 1934; Valsiner, 2014; Fossa, 2018). La historicidad de cada sujeto y la historicidad de la relación entre sujetos van a estar a la base de la construcción de la realidad. Solo la palabra-experiencia tiene sentido según la historia de la consciencia individual, y la historia como sistema relacional, de sujetos "en relación con". Los elementos de mi consciencia van a ser removidos por las nuevas experiencias o contextos solo según mi historia personal y relacional, en el fondo, según las trayectorias vitales del sujeto individual y como ente relacional. La historia de cada integrante de la relación terapéutica y la historia relacional del proceso en curso en sí, determinan la configuración del sentido de realidad.

\section{3.-El lenguaje como un acercamiento a un mundo de particularidades}

Una puerta de acceso al fenómeno de la experiencia humana resulta un desafío, en cuanto al proceso de intransferibilidad en la expresión de subjetividades. En apartados anteriores se exploró la dificultad de lograr una conexión de distintas realidades, basados en el efecto de las imposibilidades comunica- 
tivas, las cuales evidencian que las alternativas comprensivas surgen desde el sesgo interpretativo propio del observador.

En el desarrollo de la intervención en las denominadas "ciencias del espíritu" (Dilthey, 1981), se plantea la posibilidad de abordaje desde una aproximación hermenéutica a la comprensión de realidades particulares, las cuales deben contar con una dimensión comprensiva por sobre una explicativa. En esta última, solo contamos con un acercamiento a la realidad clínica, como un físico estudia la materia o bien, como quien estudia el tamaño de los ventrículos cerebrales o la medición del intelecto cuantitativamente (Jaspers, 1959).

Las ciencias del espíritu presentan un acercamiento a fenómenos propiamente humanos, asociados en primer lugar al lenguaje como determinante histórico a nivel individual, como también la cultura y sus formaciones históricas, asociando a la hermenéutica una función relacionada a la comprensión por sobre una explicación propia de las ciencias naturales (Dilthey, 1981). El acceder a los hechos de la consciencia individual, no supone un acercamiento a un mundo aislado carente de significados o bien carente de relación con los objetos del mundo circundante, se asocia a la identificación que los individuos establecen de la realidad, pudiendo acceder a esta percepción a partir del desarrollo de expresión o manifestaciones por parte del sujeto, las cuales puedan dar una posibilidad de interpretación a modo de favorecer una comprensión vivencial (Dilthey, 2000).

La influencia de Dilthey en Heidegger es trascendental, extrayendo una forma de lograr una comprensión genuina del individuo a partir de su cotidianeidad (Alltaglichkeit), lo cual constituye la forma pura de cómo el Dasein (ser en el mundo) se ve a sí mismo de manera auténtica y perceptible en su inmediatez (Heidegger, 1997). Desde la perspectiva de mutua comprensión, la emergencia de la hermenéutica se desprende como una disciplina en correlación a la vivencia particular, una interpretación adecuada propicia una posibilidad de comprensión en los citados multiversos, mas no una completa inmersión en una dimensión vivencial, debiendo considerar los alcances hermenéuticos como una aproximación de carácter interpretativo.

Pese a la imposibilidad de inmersión en la vivencia, visualizando el alcance hermenéutico solamente como una posibilidad de acercamiento interpretativo, se puede distinguir el desarrollo de una vinculación terapéutica basada en un apoyo desde lo narrativo, involucra una necesaria actitud de "ignorancia e interés" por parte del terapeuta, que establece un ejercicio basado en la suspensión teórica y en la "epoché" (acercamiento desprejuiciado), lo que resulta del todo práctico. Lograr esta posición es una compleja tarea, considerando que el desarrollo de una "curiosidad genuina" no solo puede estar fundado en un ejercicio teórico, sino también de índole vivencial (Gergen \& Gergen, 1988). Las posibilidades de cambio surgen en un proceso de aceptación que facilita la producción y expresión por parte del cliente, lo cual no significa necesariamente la conformidad estática con las premisas o narrativas establecidas por el mismo, debiendo mantener el terapeuta hermenéutico, una posición activo-participante durante el desarrollo de toda intervención (Gergen \& Kaye, 1992).

Desde los avances y beneficios, retomamos nuevamente algunas dificultades asociadas a las posibilidades de la interac- ción intersubjetiva, la cual puede verse además imposibilitada desde la perspectiva de lo que Quine (1960) denomina "la indeterminación de la traducción", la cual puede comprenderse como la dificultad en la transferencia de contenidos desde una vía lingüística, en vista de la imposibilidad de lograr una transferencia contextual que permita la comprensión por parte de un interlocutor. El clásico ejemplo graficado que expone el presente problema se observa en el encuentro entre un explorador y un nativo: ambos ante el encuentro de un conejo se asombran; el explorador llama "conejo" a dicha criatura y, por otra parte, el nativo lo denomina "gavagai". Lo que no logra comprenderse en este planteamiento es si el nativo se refiere al animal en sí, a la conejidad, una referencia respecto al tiempo o bien una referencia respecto a la caza. Esta misma imposibilidad de comprensión puede presentarse en el encuentro de dos personas que pertenezcan a dos contextos diferentes (Quine, 1968).

Similares planteamientos son extraídos de los supuestos establecidos por Wittgenstein (1953), quien en su referencia a los juegos de lenguaje (sprachspiel), indica que en determinados contextos se presentaran específicas interacciones como parte de juegos de lenguaje que permiten una interacción efectiva. Ante el encuentro con distintas instancias a nivel contextual, surge la posibilidad propia de la interpretación, mas no una inmersión que permita una fusión de horizontes ante la existencia de sesgos, tradición, cultura y experiencias impostergables en la constitución humana.

\section{4.-Una propuesta de integración comunicativa}

En los capítulos anteriormente abordados, se pudo apreciar la necesidad de establecer una vinculación de aspectos tales como el concepto de realidad y multiversos, los cuales comprenderemos como realidades particulares nutridas de lenguaje, historia y tradición, unión que surge mediante el desarrollo de una interacción dialógica que permita un encuentro de intersubjetividades. Se comentó respecto a la dificultad de lograr este encuentro intersubjetivo en el espacio de intervención psicoterapéutica, en vista de que los alcances hermenéuticos antes planteados solo podrían acercarse a fenómenos similares a la interpretación de discursos y por ello de realidades. Por ello, en el presente subcapítulo, se intentará brindar una alternativa de resolución a la conflictiva emergente en el desarrollo del presente artículo.

Para una propuesta de integración comunicativa, se valora la interacción dialógica desde una variable centrada en la articulación de "discursos que pueden ser compartidos". En efecto, es una posibilidad en el desarrollo de una relación discursiva, la generación de un propio espacio en la relación terapéutica; basándonos en la teoría de los multiversos establecida por Maturana (1997), este espacio lo denominaremos "Co-Construcción discursiva de una dimensión de realidad". Las características propias de este universo generativo de realidad a partir de una interacción intersubjetiva se ejemplifican, en el presente gráfico. 


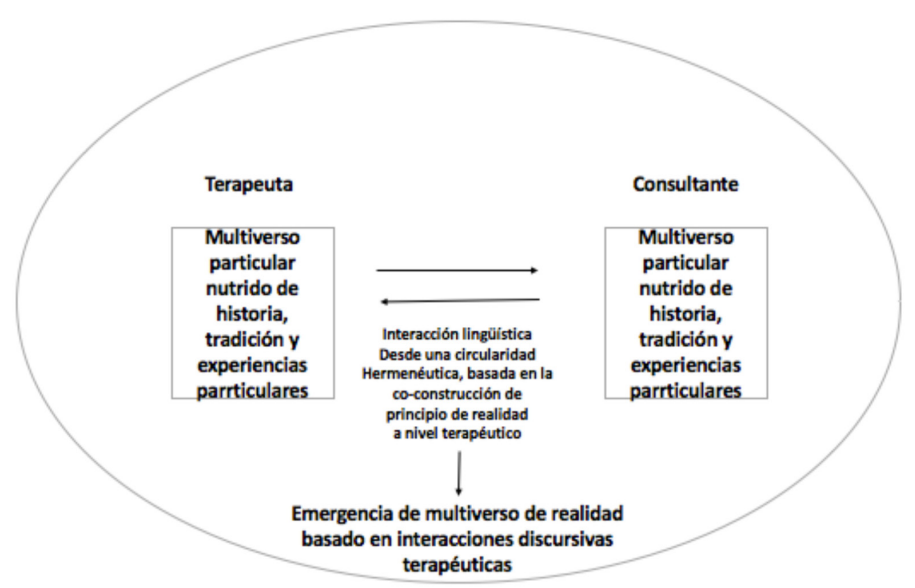

Figura 1. Gráfico de Co-Construcción discursiva de una dimensión de realidad

La figura 1 muestra el proceso de co-construcción del principio de realidad a partir de una circularidad hermenéutica. Orlinsky y Howard (1987) desarrollan un modelo genérico de proceso en psicoterapia, en el cual destacan tres aspectos: input (las variables del consultante y terapeuta antes de comenzar la psicoterapia), proceso (la relación terapéutica durante la psicoterapia) y output (las variables del consultante y terapeuta luego de terminado la terapia). Los multiversos del consultante y terapeuta en la figura $n^{\circ} 1$ son homologables a los factores de input destacados por Orlinsky y Howard (1987). Lo que agrega esta propuesta al desarrollo del modelo genérico de Orlinsky y Howard (1987), es que las variables de proceso se juegan desde una circularidad hermenéutica. En el proceso psicoterapéutico no solo se desarrolla un vínculo terapéutico que propicia el trabajo hacia las metas terapéuticas, sino que el terapeuta realiza una interpretación y re-interpretación hermenéutica de los aspectos entregados por el consultante, un permanente proceso de contraste y re-contrastación de aspectos relatados en el discurso, para así co-crear un sentido de realidad propio de esa díada en particular.

Se debe destacar el rol de Heidegger (1997) en el desarrollo de la construcción de un círculo hermenéutico, como foco de interacción dialógica propia del modelo propuesto, el cual se ve caracterizado por un encuentro en el que ambos participantes se ven nutridos de un foco de retroalimentación, no considerando conocimientos apriorísticos, lo cual vuelve esta interacción una dialógica fenomenológica hermenéutica (Heidegger, 1997). Las interacciones discursivas terapéuticas, pueden surgir a partir del apoyo de los planteamientos propios de la terapia narrativa, los cuales apuntan a un proceso operacional de deconstrucción en contacto con la figura del consultante, además de la co-identificación de una problemática (White y Epston, 1990). En el caso del modelo propuesto, esta se centrará en la conformación de un nuevo sentido de realidad, en continuidad con los procesos particulares de la figura del consultante, y a partir del multiverso generado en la interacción terapéutica. Este favorecerá la creación de alternativas de afrontamiento ante una problemática de tipo particular.

Para la generación del multiverso de "Co-construcción discursiva de una dimensión de realidad" en el espacio terapéutico, se comprometen una serie de procesos a desarrollar en el transcurso de ese espacio. A continuación, se exponen los cuatros pasos constituyentes:

1.-Mantener una suspensión teórica que garantice el surgimiento de una "epoché", en base a los supuestos de teóricos de la reducción fenomenológica de Husserl (1982).

2.-Propiciar un encuentro centrado en la interacción discursiva, la cual debe considerar un acercamiento "ingenuo" por parte del terapeuta a la problemática en cuestión.

3.-Interacción axiomática, basadas en la co-construcción dialógica de un sentido de realidad, de acuerdo a los planteamientos expuestos en el construccionismo social de Kenneth Gergen (2007), como en los supuestos del dinamismo discursivo de Linell (1998), el cual expone que la temática a abordar dependerá de la construcción discursiva de los actores participantes en una interacción determinada.

4.-Definición conjunta del "sentido de realidad o realidades", co-construidas en el proceso de intervención psicoterapéutica, las cuales garanticen un nuevo sentido que favorezca la apropiación por parte del consultante de un nuevo enfoque ante la problemática inicial que motivó su participación en el proceso terapéutico.

La presente propuesta de integración comunicativa responde de manera clara a una propuesta dentro de la línea fenomenológica hermenéutica, la cual pretende garantizar una vinculación desprejuiciada por parte del terapeuta, que comprometa el desarrollo de argumentos y procesos discursivos favorecedores de nuevos horizontes de interacción. Se espera que estos permitan una apropiación por parte del consultante de su propia problemática, como también, la generación de una vinculación que facilite una interacción que trascienda las esferas intersubjetivas, al desarrollar "códigos" particulares entre la relación terapeuta-consultante.

\section{Discusión}

La propuesta de integración comunicativa compromete un rol activo desde una dimensión lingüística de percepción y definición de la realidad. Es mediante la generación de un modelo de abordaje sobre una interacción discursiva, que se busca favorecer el desarrollo de interacciones que trasciendan dimensiones subjetivas, generando un espacio compartido y propio entre la figura de terapeuta y consultante. Entre los desafíos que destacan de esta co-construcción de sentido de realidad entre estos dos actores, resalta la necesidad de contar con terapeutas que puedan mantener una visión respecto al sentido de realidad, considerando una visión lingüística basada en los planteamientos establecidos por Kenneth Gergen (2007). Tal autor destaca el valor del lenguaje en la construcción de posibles realidades, desde su teoría de construccionismo social.

Surge también la necesidad de que este encuentro de miradas respete la visión particular del consultante, ante lo que una suspensión teórica resulta una acción relevante, permitiendo un acercamiento fenomenológico. Es menester referir que la instancia asociada a generar una co-construcción discursiva del sentido de realidad con el consultante, representa un 
desafío fenomenológico - hermenéutico, debiendo primar un dominio epistemológico desde los planteamientos que revisten una visión ontológica respetuosa de un ser perteneciente a un mundo con historia, tradición y lenguaje.

En la presente propuesta no queda resuelta en mayor detalle, la participación o rol del consultante, debiendo profundizar las posibles posiciones de dicha figura en futuras investigaciones, sin que ello represente una posición apriorística en la percepción del consultante como un ente predecible o de acciones predeterminadas.

\section{Referencias}

Cornejo, C. (2012). Contrasting Vygotsky's and Bakhtin's approaches to consciousness. Culture \& Psychology, 18(1), 109-120.

Dilthey, W. (1981). Introducción a las ciencias del espíritu. Madrid: Alianza Editorial.

Dilthey, W. (2000). Dos escritos sobre hermenéutica: el surgimiento de la hermenéutica y los esbozos para una crítica de la razón histórica. Madrid: Istmo.

Fossa, P. (2018). The problem of reality in psychology: Revisiting the reality concept in Oswald Külpe. Human Arenas, 1(1), 86-96.

Gadamer, H. G. (1982). Truth and Method. New York: Crossroads

Gadamer, H.G. (2010). Verdad y método II. Salamanca: Sígueme.

Gergen, K., Gergen M. (1988). Narrative and the self as relationship. Advances in Experimental Social Psychology, 21, 17-56.

Gergen, K. (2007). Construccionismo Social. Aportes para el debate y la práctica. Bogotá: Ediciones Uniandes.

Gergen, K., Kaye, J. (1992). Beyond narrative in the negotiation of human meaning. London: Sage.

Heidegger, M. (1997). Ser y Tiempo. Santiago de Chile: Editorial Universitaria.

Husserl, E. (1982). La idea de la fenomenología. Cinco lecciones, Madrid: FCE. Traducción de Miguel García-Baró.

Jaspers, K. (1959). Razón y Existencia. Buenos Aires: Editorial Nova.

Jauss, H.R. (1977). Ästhetische Erfahrung und literarische Hermeneutik. München: Fink Verlag München.

Kenny, V. (1989). Life, the multiverse and everything; An introduction to the ideas of Humberto Maturana, 'Self-Organisation in Psychotherapy.' Heidelberg: Springer Verlag.

Maturana, H. (1978). Biology of Language: The epistemology of reality. In G. Miller \& E. Lenneberg (Eds.), Psychology and Biology of Language and Thought (pp.28-62). New York: Academic Press.

Maturana, H. (1987). Everything is said by an Observer. In: Gaia, a way of Knowing. Political implications of the New Biology. I. Thompson. New York: Lindsfarne Press.

Maturana, H. (1988). Reality: The search for objectivity or the quest for a compelling argument. The Irish Journal of Psychology, 9(1), 25-82.

Maturana, H. (1997). La objetividad. Un argumento para obligar. Santiago: Dolmen Ediciones.

Maturana, H. \& Kurt, L. (1994). Reflexiones y Conversaciones. Cordova: Ediciones FUPALI.
Mook, B. (2010). Hermeneutic Phenomenology and Psychotherapy. Les Collectifs du Cirp, 1 (édition spéciale), 209-222.

Linell, P. (1998). Approaching Dialogue: Talk, Interaction and Contexts in Dialogical Perspectives. Amsterdam: John Benjamins.

Orlinsky, D.E. \& Howard, K. I. (1987). A Generic Model of Psychotherapy. Journal of Integrative and Eclectic Psychotherapy, 6, 6-27.

Quine, W.V.O. (1960). Word and Object. Massachusetts: Mit Press.

Quine, W.V.O. (1968). Ontological Relativity. Ontological Relativity and Other Essays. New York: Columbia University Press.

Valsiner, J. (2014). An invitation to Cultural Psychology. London: SAGE Publications.

Valsiner, J. \& Van der Veer, R. (2000). The social mind: Construction of the idea. USA: Cambridge University Press.

Vygotsky, L. (1934). Pensamiento y Lenguaje. Madrid: Paidos.

White, M \& Epston, D. (1990). Medios narrativos para fines terapéuticos, Buenos Aires: Paidos.

Wittgenstein, L. (1953). Philosophical investigations. New York 